\title{
Stendhal et les choses de la nature
}

\section{Annalisa Bottacin}

\section{(2) OpenEdition}

\section{Journals}

\section{Edizione digitale}

URL: https://journals.openedition.org/studifrancesi/11830

DOI: 10.4000/studifrancesi. 11830

ISSN: 2427-5856

\section{Editore}

Rosenberg \& Sellier

\section{Edizione cartacea}

Data di pubblicazione: 1 avril 2018

Paginazione: 145-146

ISSN: 0039-2944

\section{Notizia bibliografica digitale}

Annalisa Bottacin, «Stendhal et les choses de la nature», Studi Francesi [Online], 184 (LXII | I) | 2018,

online dal 03 juillet 2018, consultato il 16 novembre 2021. URL: http://journals.openedition.org/ studifrancesi/11830 ; DOI: https://doi.org/10.4000/studifrancesi.11830

Questo documento è stato generato automaticamente il 16 novembre 2021.

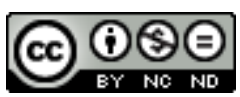

Studi Francesi è distribuita con Licenza Creative Commons Attribuzione - Non commerciale - Non opere derivate 4.0 Internazionale. 


\title{
Stendhal et les choses de la nature
}

\author{
Annalisa Bottacin
}

\section{NOTIZIA}

Stendhal et les choses de la nature, Actes du colloque de Paris-INHA, 26-27 mars 2010, organisé par «Stendhal Aujourd'hui», études réunies et présentées par Michel ARRous, Paris, Eurédit, 2017, 168 pp.

1 Il titolo del presente volume, che raccoglie gli atti di un interessante convegno svoltosi all'Institut National d'Histoire de l'Art di Parigi, viene parzialmente estrapolato da una pagina dei Mémoires d'un touriste, contrassegnata da una riflessione di Stendhal intorno al concetto di pittoresque, un'espressione che appare chiaramente sul piano delle scelte lessicali del grenoblese. Nell'opera stendhaliana l'idea di paesaggio è più suggerita che descritta, come ben rileva Michel ARRous nella Présentation (pp. VII-X), ma non mancano certo equivalenze tra sensazione e spazio paesaggistico. Il paesaggio è da considerarsi uno dei temi classici non solo negli studi geografici; in effetti, nell'accezione più corrente, indica il settore di un territorio che la natura presenta all'osservatore. Trattandosi di termine polisemico è interessante precisare dunque cosa intenda Stendhal per paesaggio e la funzione che esso assume nei suoi romanzi. La natura, vista attraverso gli occhi dei suoi personaggi, viene trasformata dall'azione e dallo sguardo. Sovente l'eroe, in special modo nel Rouge e nella Chartreuse, raggiunge la perfetta compiutezza solo nella comunione con un panorama che risponda più specificatamente ai suoi sentimenti e alle sue aspirazioni. La funzione estetica del paesaggio è determinante in Henri Beyle, come ben viene evidenziato in questi atti. MarieFrançoise GUINOISEAU in Voyages stendhaliens et paysages, par monts et vallées, lacs et forêts, de 1800 à 1836. Leçon de choses (pp. 1-20) ne dà rilevante esempio; un'analisi dei viaggi in Italia del giovane Beyle evidenziano infatti la funzione essenziale della sua idea di paesaggio. Sono tracce che emergono da appunti, piccole annotazioni, ma che una volta legati insieme producono l'effetto voluto, ricollocandosi sulla pagina e producendo nel personaggio inverosimili complicità che lo coinvolgono intimamente. Prosegue nella medesima indagine, in una comparazione con Balzac, Max ANDRÉoLI (Arbres et Forêts, che 
nell'indice diventa Forêts et arbres chez Stendhal et Balzac, pp. 31-49) che espone, con una serie di esemplificazioni, le varie divergenze tra i due scrittori, esordendo con alcune pagine degne di nota sul concetto di nature; Andrée MANSAU in Pyrénées, «ubi es?» (pp. 51-59) organizza il suo discorso seguendo le linee di diramazione e di convergenza tra Stendhal da un lato e dall'altro Victor Hugo e George Sand, nella comune visione del paesaggio dei Pirenei, la cui reazione, descritta dall'autore del Rouge nei Mémoires d'un touriste, poco attratto da cime elevate, si distacca nettamente da quella degli altri due romanzieri. Élisabeth SCHEELE in Guerre et nature chez Stendhal et Shakespeare (pp. 21-30), riflettendo in primo luogo sull'ammirazione profonda di Stendhal per il drammaturgo britannico, produce una serie di raffronti tra alcuni passaggi di tragedie quali Macbeth, Hamlet e Antoine et Cléopâtre, in cui la natura svolge un ruolo di spicco, e alcune pagine stendhaliane dedicate ai viaggi in Italia, sottolineando come l'esplorazione paesaggistica contempli una forza produttiva di minuziosi ed emblematici stati di essere. Encarnación MEDINA ARJONA ci porta in campo artistico in un raffinato studio dedicato al processo che lega Stendhal a Claude Lorrain ("À l'ombre de beaux arbres», ou la lumière de Claude Lorrain chez Stendhal, pp.61-79), un artista che viene sovente citato nell'opus stendhaliano. Henri Beyle ammira profondamente la concezione paesistica classica o storica del grande pittore barocco lorenese, in particolar modo nella idealizzazione del paesaggio della campagna romana. Traendo spunto dai riferimenti pittorici presenti nei romanzi e nei racconti dell'ultimo periodo, Janine GALLANT, come ben si può rilevare dal titolo del suo contributo, Les choses de la nature et les choses de la peinture: le mariage romanesque des dernières années (pp. 81-90), si sofferma in modo chiaro e preciso sul rapporto Nature e Art nel senso più lato, un legame fondamentale in Stendhal. Sin dalle prime lettere inviate alla sorella Pauline, un giovane Beyle dimostra "une double sensibilité à la nature et aux arts», che si tramuta nella scrittura in un continuo legame in particolar modo tra natura e pittura, cosicché questo procedimento romanesque genera un'estetica globale delle sensazioni. Nel terzo libro del Capitale, Karl Marx soffermandosi sul tema di capitale-profitto, capitale-interesse, terra-rendita fondiaria, lavoro-salario presenta la mistificazione del modo di produzione capitalistico: è il mondo perverso, deformato e sconvolto in cui si aggirano i fantasmi di Monsieur le Capital et Madame la Terre, immagini che sono maschere, strumenti di una propaganda mistificante. Ispirandosi alla celebre formula del filosofo ed economista tedesco e alla sua teoria dell'illusionismo sociale, Francesco SPANDRI in "Monsieur le Capital et Madame la Terre" (pp. 91-107) propone un'interessante rilettura di Armance, romanzo della terra perduta e dell'intrusione del capitale. Pierrette PAVET pone la sua attenzione su un altro aspetto del rapporto di Stendhal con il fatto naturale, per altro poco studiato, quello della «laideur des choses» che ben si accompagna alla «laideur morale» (Stendhal et la laideur des choses, pp. 109-118). Questo nasce dal realismo dello scrittore che, accanto a spettacoli di grande bellezza o meglio al sublime, non manca di usare corsivi nell'uso di segni verbali quali puant, sale, ecc.; da non tralasciare per altro che, a dire di Michel Crouzet, «le laid, c'est le réel». Le bellezze naturali ritornano nel bell'articolo di Jean-Jacques нАмм (Nominalisme et nature: bois et forêts des romans et nouvelles stendhaliens che nell'Index diventa Nominalisme et nature: bois et forêts stendhaliens, pp. 119-129); boschi e foreste sono luoghi di incontro, luoghi del benessere, nello studio del legame tra gli individui e il loro universo. Il critico sottolinea come nell'opera stendhaliana si presentino quali «lieux individuels visités par des consciences individuelles». In conclusione Michel CROUZET ci investe della sua profonda conoscenza di Stendhal con un corposo e dotto contributo che dallo stesso titolo 
testimonia l'autorità dell'argomento. Titolo breve ma efficace, Le ciel et la terre chez Stendhal (pp.131-66), è un'opposizione su cui l'eminente stendhaliano si era già cimentato in Nature et société chez Stendhal. La révolte romantique (1985). Partendo dal presupposto della continuità in Stendhal di termini religiosi o vicini al campo della religiosità, Crouzet intende analizzare l'antinomia ciel-terre, sfiorando di continuo il limite tra mondo naturale e mondo soprannaturale, portando quale referente l'uso in Stendhal di un immenso lessico (in cui non si può esimersi dall'inserire il lemma âme, distintivo dell'insieme delle passioni), che comporta un repertorio di espressioni religiose, che tuttavia sono usate in un contesto profano. Crouzet evidenzia, appoggiandosi a un insieme di assai esplicative esemplificazioni tratte da testi stendhaliani, come per lo scrittore la tematica del sacro emerga soprattutto quale residuo effettivo del sistema di pensiero e si esprima attraverso una costellazione organica di senso contario rispetto a quella della totalità. 